\title{
Association of mentally-passive and mentally-active sedentary behaviours during adolescence with psychological distress during adulthood: a 26-year prospective study
}

André O. Werneck ${ }^{1,2}$, Erin Hoare ${ }^{2,3}$, Brendon Stubbs ${ }^{4}$, Esther M. F. van Sluijs ${ }^{2}$ and Kirsten Corder ${ }^{2}$.

1. Universidade Estadual Paulista "Júlio de Mesquita Filho" (UNESP), Presidente Prudente, Brazil. 2. MRC Epidemiology Unit and Centre for Diet and Activity Research (CEDAR), University of Cambridge, Cambridge, UK. 3. Deakin University, Melbourne, Victoria 3004, Australia. 4.

King's College London, De Crespigny Park, London, UK. E-mail: andre.o.werneck@unesp.br

\section{Introduction}

- Behaviours as physical activity associated with depressive symptoms.

- Sedentary behaviour associated with elevated depressive symptoms.

- Different types of sedentary behaviour can have different associations with depressive symptoms.

- Sedentary behaviours can be classified as mentally-active and mentally-passive sedentary behaviour.

- Reduced evidence (only among middle-aged adults).

- Potential mediators unexplored (including behaviours, physical and psychological factors)

\section{Objective}

To analyse the association between mentally-active and mentallypassive sedentary behaviour during adolescence and psychological distress during adulthood, and to examine potential mediators

\section{Methods}

- 1970 British Cohort Study ( $\mathrm{n}=1,787$ - 706 men).

- During adolescence (16 years):

Frequency of reading newspaper and magazine during previous week.

Time watching TV, movies, reading books and doing homework.

mentally-passive (TV-viewing, watching movies - 3h/day or more) and mentally-active (reading books, newspapers, and magazines, homework, playing computer games - two or more) sedentary behaviour created.

Psychological distress (malaise inventory) and organized sports participation.

- During adulthood (42 years):

TV-viewing, psychological distress (malaise inventory), smoking, pain perception, physical limitations, self-rated health, were considered as potential mediators (all treated as binary)

- Covariates: Sex, employment status (42y), education, sports participation at 16y and psychological distress at 16y.

\section{Statistics}

Frequencies and estimated $95 \%$ confidence intervals

Logistic regression models

- Mediation models (Karlsson-Holm-Breen)

Decomposes total effect into direct effect and indirect effect as well as provide \% meditation using logistic regressions.

- Theoretical model presented on Figure 1.

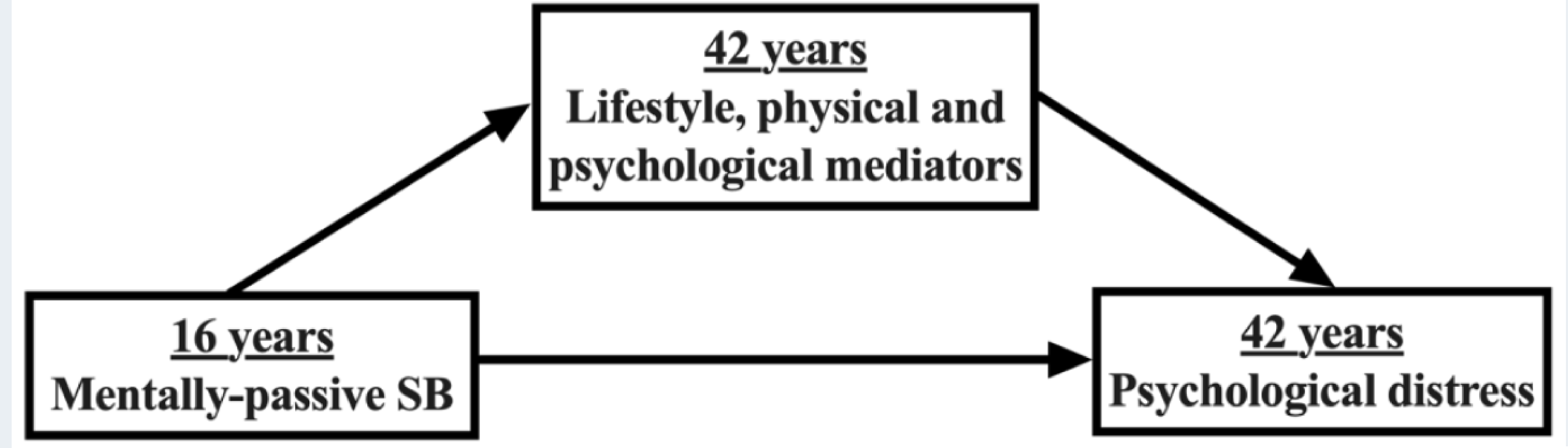

Figure 1. Theoretical model. Note. SB, sedentary behaviour

\section{Results}

Table 1. Association of sedentary behaviour during adolescence and elevated psychological distress symptoms (Malaise Inventory) at adolescence (cross-sectional) and adulthood (prospective).

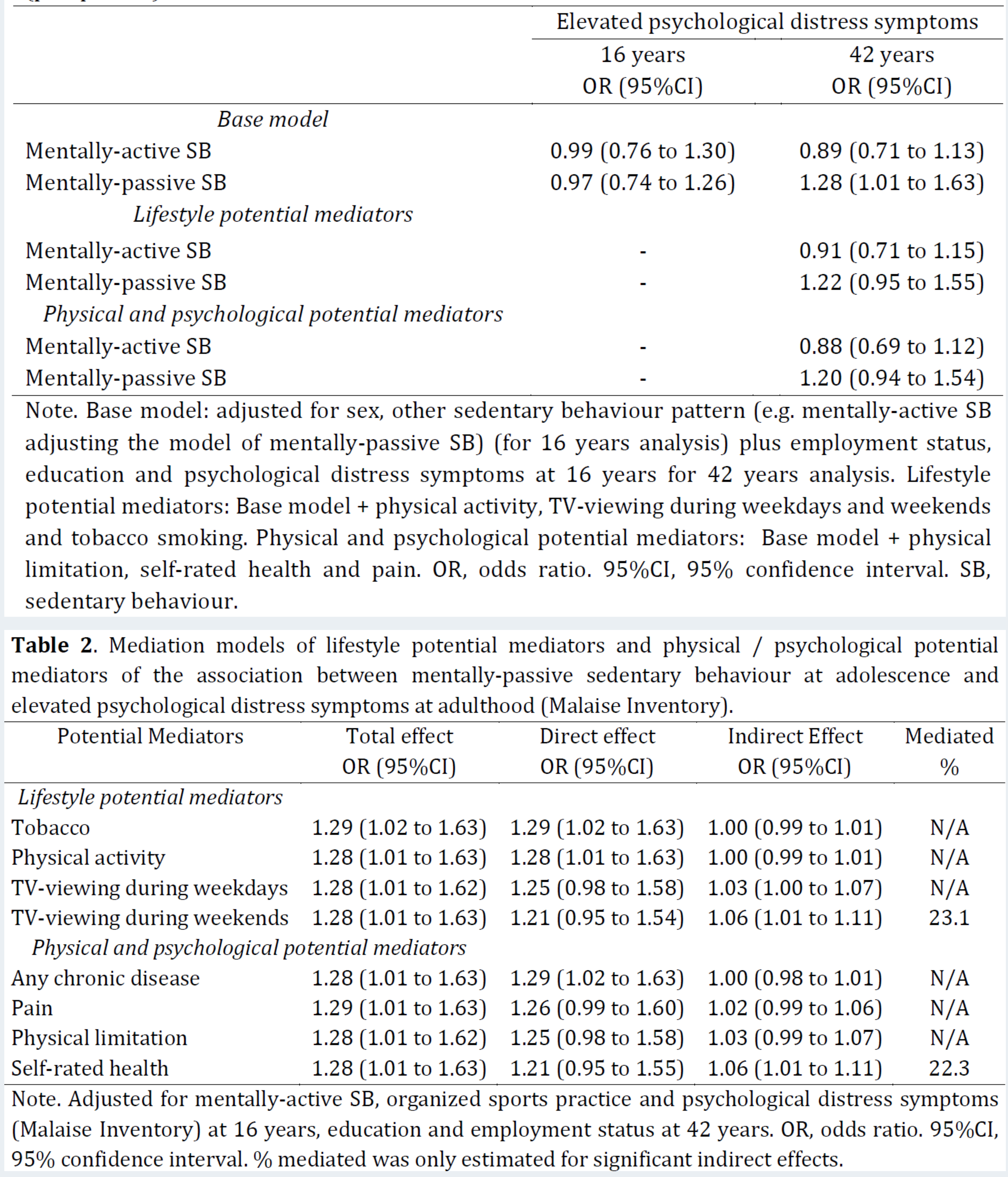

\section{Conclusion}

Weekend TV-viewing and self-rated health are potential mediators of the harmful association between mentally-passive sedentary behaviour in adolescence and psychological distress during adulthood. Interventions to improve mental health could focus on mentally-passive behaviours instead of total sedentary behaviour and also potential mediators during adulthood.

\section{Acknowledgements}

We thank to São Paulo Research Foundation for the scholarship of André O. Werneck (process 2018/19183-1). 